\title{
miR-181c promotes proliferation via suppressing PTEN expression in inflammatory breast cancer
}

\author{
WEN-LONG ZHANG and JIAN-HUA ZHANG
}

General Surgery Department, The Second Artillery General Hospital of PLA, Beijing 100088, P.R. China

Received November 30, 2014; Accepted January 21, 2015

DOI: $10.3892 / \mathrm{ijo} .2015 .2896$

\begin{abstract}
Primary inflammatory breast cancer (IBC) accounts for $\sim 6 \%$ of new breast cancer cases. Even with multimodality treatment, the 5-year disease-free survival is $<45 \%$, thus making IBC the most deadly form of locally advanced breast cancer. Better understanding of the pathogenesis of IBC is essential to the design of effective therapy. We found that miR-181c was upregulated in IBC, implying that it could be a useful prognostic marker for IBC and a novel therapeutic target for the intervention of disease. Elucidating why the gene is overexpressed and how to downregulate it will help us to further understand the pathogenesis and progression of the disease and offer new targets for therapies. In this study, we showed that miR-181c as an oncogene promoted proliferation and it inhibited PTEN protein expression by targeting 3'-UTR of PTEN mRNA in IBC SUM149 cells. Moreover, PTEN was not only downregulated in IBC, but also inhibited proliferation in SUM149 cells and introduction of PTEN cDNA lacking the predicted sites of 3'-UTR abrogated miR-181c cellular function, suggesting that miR-181c inhibited proliferation by downregulating PTEN expression in IBC. Thus, targeting miR-181c and restoration of PTEN can be used in conjunction with other therapies to prevent progression of IBC.
\end{abstract}

\section{Introduction}

Breast cancer is a major cause of morbidity and mortality in the world (1). A rare ( $6 \%$ of all cases) but often lethal form of locally advanced breast cancer is inflammatory breast cancer (IBC). IBC is a form of locally advanced breast cancer (LABC) that was first described by Lee and Tannenbaum in 1924 (2). The unique clinical and pathologic syndrome and discouraging outcome make IBC a very distinct form of breast carcinoma (reviewed in refs. 3,4). IBC frequently displays high histologic grade, negativity of hormone receptors, neoangiogenenesis and tissue invasive-

Correspondence to: Dr Jian-Hua Zhang, General Surgery Department, The Second Artillery General Hospital of PLA, Beijing 100088, P.R. China

E-mail: jianhua_zhang_pla@qq.com

Key words: inflammatory breast cancer, miR-181c, PTEN ness, and an aggressive clinical behavior with high frequency of axillary lymph node involvement at diagnosis and, in $~ 35 \%$ of cases, distant metastasis (3). Despite advances in the multidisciplinary treatment, the prognosis is much less favorable than for non-IBC, with a 5-year survival ranging from 30 to $50 \%(3,5)$. Because of its relative scarcity and difficulty for obtaining diagnostic samples of sufficient size, the molecular basis of IBC still waits clarification (4).

MicroRNAs (miRNAs/miRs) are a class of non-coding RNAs able to regulate gene expression at the post-transcriptional level, by binding to the 3'-untranslated region of target messenger RNAs (mRNAs) through partial sequence homology, and causing a block of translation and/or mRNA degradation (6-8). Aberrant miRNA expression has been linked to diseases, including cancer and it has been found implicated in a multitude of cellular processes including proliferation, differentiation, migration, and apoptosis (9-14). Evidence for the causal involvement of miRNAs in breast cancer comes from many sources. For example, invasion and metastasis of breast cancer was initiated by miR-10b (15); microRNA miR-21 overexpression in human breast cancer is associated with advanced clinical stage, lymph node metastasis and patient poor prognosis and PDCD4 is an important functional target of miR-21 in breast cancer cells $(16,17)$; miR-31 inhibits breast cancer metastasis (18); downregulation of miRNA-200c links breast cancer stem cells with normal stem cells (19); microRNA-221/222 confers tamoxifen resistance in breast cancer by targeting $\mathrm{p} 27^{\mathrm{Kip} 1}$ (20). miRNAs play important roles in breast cancer, but roles of miRNA in inflammatory breast cancer are still scarely reported.

In this study, first, we confirmed that miR-181c expression was upregulated in inflammatory breast cancer tissues, compared with adjacent normal tissues and showed that it could promote proliferation in IBC cells. microRNAs (miRNAs or mirs) are short non-coding RNAs that regulate the target mRNAs by binding mostly to the $3^{\prime}$ untranslated region (3'-UTR), inducing either translational repression or the degradation of the target (6-8). We screened target genes of miR-181c by TargetScan which is a commonly used prediction algorithm. We found that miR-181c could target 3'-UTR of PTEN mRNA. Following studies confirmed that miR-181c downregulated PTEN expression in inflammatory breast cancer cells by targeting its 3'-UTR. Finally, we confirmed that PTEN was downregulated in inflammatory breast cancer tissues as well as it inhibited proliferation in the 
cells. Introduction of PTEN cDNA lacking predicted sites of 3'-UTR abrogated miR-181c cellular function. Thus, we reason that due to the overexpression of miR-181c gene in inflammatory breast cancer, the tumor suppressive gene PTEN is downregulated and then downregulation of the gene further promotes proliferation.

\section{Materials and methods}

Inflammatory breast cancer tissues, SUM149 cells, PTEN expressing plasmids/empty vectors and transfection experiments. Six human inflammatory breast cancer tissues were from the Second Artillery General Hospital of PLA and Tongji Hospital. The use of human tissue samples followed internationally recognised guidelines as well as local and national regulations. Research carried out on humans follow international and national regulations. Medical ethics committee of our hospital approved the experiments undertaken. Informed consent was obtained from each individual. Inflammatory breast cancer cell line SUM149 was kindly donated by Dr Robert Kagan (Nagasaki University, Japan). Briefly, the SUM149 cell line was developed from a primary IBC tumor and grown in 5\% FBS-supplemented Ham's F-12 medium containing insulin and hydrocortisone. PTEN expressing plasmids and empty vectors were donated by X. Yao (The Pennsylvania State University). For transfection experiments, the cells were cultured in serum-free medium without antibiotics at $60 \%$ confluence for $24 \mathrm{~h}$, and then transfected with transfection reagent (Lipofectamine 2000, Invitrogen) according to the manufacturer's instructions. After incubation for $6 \mathrm{~h}$, the medium was removed and replaced with normal culture medium for $48 \mathrm{~h}$.

miRNA precursors, anti-miRNA oligonucleotides. The miR-181c miRNA precursor (pre-miR-181c) and a control precursor (control miR) were purchased from Ambion, Inc. The locked nucleic acid (LNA)-modified oligonucleotide inhibitors (anti-miR-181c) used for miRNA knockdown and scramble were purchased from Exiqon.

miRNA microarray. Total RNA from cultured cells, with efficient recovery of small RNAs, was isolated using the mirVana miRNA Isolation kit (Ambion, Austin, TX, USA). cRNA for each sample was synthesized by using 3' IVT Express kit (Affymetrix, Santa Clara, CA, USA) according to the manufacturer's protocols. The purified cRNA was fragmented by incubation in fragmentation buffer (provided in the $3^{\prime} \mathrm{IVT}$ express kit) at $95^{\circ} \mathrm{C}$ for $35 \mathrm{~min}$ and chilled on ice. The fragmented labeled cRNA was applied to MicroRNA2.0 array (Affymetrix) and hybridized in Genechip hybridization oven 640 (Affymetrix) at $45^{\circ} \mathrm{C}$ for $18 \mathrm{~h}$. After washing and staining in Genechip fluidics station 450 (Affymetrix), the arrays were scanned by using Genechip scanner 3000 (Affymetrix). The gene expressions levels of samples were normalized and compared by using Partek GS 6.5 (Partek, Inc., St. Louis, MO, USA). Average-linkage hierarchical clustering of the data was applied by using the Cluster (Eisen et al, Stanford University, CA, USA; and the results were displayed by using TreeView (Eisen et al, Stanford University, CA, USA; http://rana.lbl.gov).
Real-time PCR for miRNA. Total RNA from cultured cells, with efficient recovery of small RNAs, was isolated using the mirVana miRNA Isolation kit (Ambion). Detection of the mature form of miRNAs was performed using the mirVana qRT-PCR miRNA Detection kit, according to the manufacturer's instructions (Ambion). The U6 small nuclear RNA was used as an internal control.

Colony formation. For colony formation assay, cells were transfected for $24 \mathrm{~h}$, and then seeded in a 6-well plate. FBS $(0.2 \mathrm{ml})$ was added per well on day 5. After 9-10 days incubation, plates were washed with PBS and stained with $0.1 \%$ of crystal violet. Colonies with $>50$ cells were manually counted. Plating efficiency was calculated by dividing the number of colonies formed in the treated group by that in control.

\section{3-(4, 5-Dimethyl-2-thiazolyl)-2, 5-diphenyl-2H-tetrazolium} bromide (MTT) assay. Cells seeded on 96-well plates, were stained at indicated time point with $100 \mathrm{ml}$ sterile MTT dye $\left(0.5 \mathrm{mg} / \mathrm{ml}\right.$, Sigma, St. Louis, MO, USA) for $4 \mathrm{~h}$ at $37^{\circ} \mathrm{C}$, followed by removal of the culture medium and addition of $150 \mathrm{ml}$ of dimethyl sulphoxide (DMSO) (Sigma, St. Louis, MO, USA). The absorbance was measured at $570 \mathrm{~nm}$, with $655 \mathrm{~nm}$ as the reference wavelength.

Cell cycle analysis. SUM149 cells (8.0×10 5 cells) were seeded into a $100-\mathrm{mm}$ culture plate and allowed to attach overnight. The cells were transfected with pre-miR-181c/control miR or anti-miR-181c/scramble or PTEN expressing plasmids/ empty vectors for $24 \mathrm{~h}$, washed twice with $\mathrm{NaCl} / \mathrm{Pi}$, and then centrifuged at $200 \mathrm{~g}$ at room temperature. The pellet was resuspended in $1 \mathrm{ml}$ cold $\mathrm{NaCl} / \mathrm{Pi}$ and fixed in $70 \%$ ethanol for $\geq 12 \mathrm{~h}$ at $4^{\circ} \mathrm{C}$. The fixed cells were incubated with $100 \mu \mathrm{l}$ DNase-free RNaseA $(200 \mu \mathrm{g} / \mathrm{ml})$ for $30 \mathrm{~min}$ at $37^{\circ} \mathrm{C}$ and then $1 \mathrm{mg} / \mathrm{ml}$ propidium iodide was added. The stained cells were analyzed using a fluorescence-activated cell sorter (BD Accuri C6; BD Biosciences, Ann Arbor, MI, USA). The percentages of cells in the G1, S and G2/M phases of the cell cycle were determined using Cell Quest Pro software (FlowJo, Ashland, OR, USA).

Bromodeoxyuridine labeling and immunofluorescence. Cells grown on coverslips (Fisher, Pittsburgh, PA, USA) were incubated with bromodeoxyuridine (BrdUrd) for $1 \mathrm{~h}$ and stained with anti-BrdUrd antibody (Upstate, Temecula, CA, USA) according to the manufacturer's instructions. Gray level images were acquired under a laser scanning microscope (Axioskop 2 plus, Carl Zeiss Co. Ltd., Jena, Germany).

Western blot analysis. Western blot analysis was performed as described before (21). Mainly, after incubation with primary antibody anti-CDK4 (1:500; Abcam, Cambridge, MA, USA), anti-PCNA (1:500; Abcam), anti-c-myc (1:500; Abcam), anti-p21 (1:500; Abcam), anti-p53 (1:500; Abcam), anti-PTEN $(1: 500 ;$ Abcam) and anti- $\beta$-actin $(1: 500 ;$ Abcam) overnight at $4^{\circ} \mathrm{C}$, IRDye $\mathrm{TM}_{-}^{\mathrm{TM}} 800$ conjugated anti-rabbit secondary antibodies (Li-COR, Biosciences, Lincoln, NE, USA) were used for $30 \mathrm{~min}$ at room temperature. The specific proteins were visualized by Odyssey ${ }^{\mathrm{TM}}$ Infrared Imaging System (Gene Co., Lincoln, NE, USA). 
A
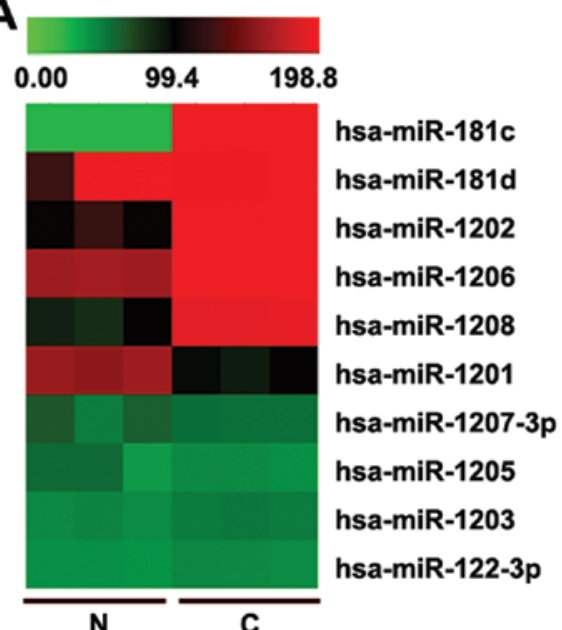

B

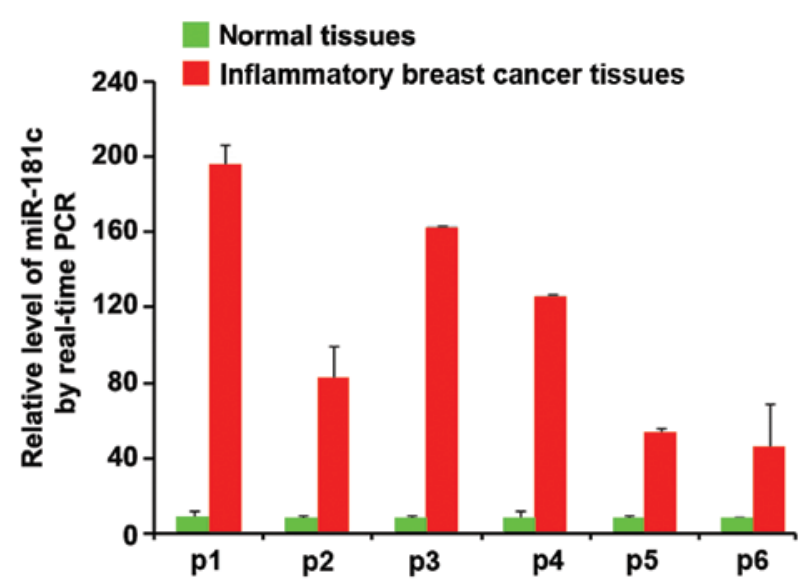

Figure 1. miR-181c expression is upregulated in inflammatory breast cancer. (A) Partial heat map of miRNA microarray analysis of inflammatory breast cancer tissues, compared with adjacent normal tissues. $n=3$. (B) Real-time PCR for miR-181c in inflammatory breast cancer tissues, compared with adjacent normal tissues. $n=6$.

Methods of bioinformatics. The analysis of potential microRNA target sites using a common prediction algorithm. TargetScan (http://www.targetscan.org).

Immunofluorescence analyses. For immunofluorescence analyses, SUM149 cells were plated on glass coverslips in 6-well plates and transfected with $30 \mathrm{nM}$ pre-miR-181c or control miR. At $36 \mathrm{~h}$ after transfection, coverslips were stained with the anti-PTEN antibodies. Alexa Fluor 488 goat anti-mouse IgG antibody or goat anti-rabbit IgG antibody was used as secondary antibody (Invitrogen, Carlsbad, CA, USA). Coverslips were counterstained with DAPI (InvitrogenMolecular Probes, Eugene, OR, USA) for visualization of nuclei. Microscopic analysis was performed with a confocal laser-scanning microscope (Leica Microsystems, Bensheim, Germany). Fluorescence intensities were measured in a few viewing areas for 200-300 cells per coverslip and analyzed using ImageJ $1.37 \mathrm{v}$ software (http://rsb.info.nih.gov/ij/index. html).

Reverse-transcription polymerase chain reaction (RT-PCR) and quantitative real-time RT-PCR ( $R R T-P C R)$ for PTEN. Total RNA was isolated from cells using TRIzol reagent (Invitrogen). First-strand cDNA was synthesized from the total RNA using M-MLV reverse transcriptase (Promega, Madison, WI, USA) and random hexamer primers (Sangon, Shanghai, China). The thermal cycle profile was as follows: denaturation for $30 \mathrm{sec}$ at $95^{\circ} \mathrm{C}$, annealing for $45 \mathrm{sec}$ at $52-58^{\circ} \mathrm{C}$ depending on the primers used, and extension for $45 \mathrm{sec}$ at $72^{\circ} \mathrm{C}$. PCR products were visualized on $2 \%$ agarose gels stained with ethidium bromide under UV transillumination. qRT-PCR was done with a Power SYBR Green PCR Master Mix (Applied Biosystems, Carlsbad, CA, USA) according to the manufacturer's protocol. The primer sequences for PTEN: 5'-AATTT TTAAAGGCACAAGAGG-3' and 5'-TCCAGGAAGAGGA AAGGA-3'.

Luciferase reporter assay. The luciferase reporter plasmids were from Tiangene (Tianjin, China). The 3' untranslated region
(3'-UTR) of human PTEN mRNA was inserted into pRL-TK (Promega) using PCR-generated fragment. Site-directed mutagenesis of the miR-181c target-site in the PTEN-3'-UTR was carried out using Quik change-mutagenesis kit (Stratagene, Heidelberg, Germany), with PTEN-WT-luc as a template. For reporter assays, SUM149 cells was transiently transfected with WT or mutant reporter plasmid and miR or anti-miR (as indicated in Fig. 4F and G) using Lipofectamine 2000 (Invitrogen). Reporter assays were performed $36 \mathrm{~h}$ post-transfection using the Dual-luciferase assay system (Promega), normalized for transfection efficiency by cotransfected Renilla-luciferase.

\section{Results}

miR-181c expression is upregulated in inflammatory breast cancer tissues. In an attempt to identify the miRNAs expression between inflammatory breast cancer tissues and adjacent normal tissues, we performed miRNA profiling in cancer tissues and normal tissues. RNAs isolated from 3 pairs of breast cancer tissues and normal tissues were hybridized to a custom miRNA microarray platform containing 2100 miRNAs. After three hybridizations, quantification, and normalization, a dozen miRNAs, especially miR-181c was elevated in inflammatory breast cancer $>190$-fold, compared with normal tissues (Fig. 1A). Consistent with the miRNA microarray data, real-time PCR analysis revealed the expression of miR-181c in 6 pairs of IBC tissues were significantly elevated, compared with adjacent normal tissues (Fig. 1B). The data implied that miR-181c could be an oncogene in inflammatory breast cancer.

miR-181c promotes proliferation in inflammatory breast cancer cells. In an attempt to identify the role of miR-181c in regulating proliferation of SUM149 cells, cells were transfected with pre-miR-181c. After stable transfection, miR-181c expression was detected by real-time PCR and the colony formation of SUM149 cells were tested by colony formation assay. The results showed that exogenous pre-miR-181c stably upregulated miR-181c expression in SUM149 cells (Fig. 2A) and overexpression of miR-181c significantly increased colony 
A

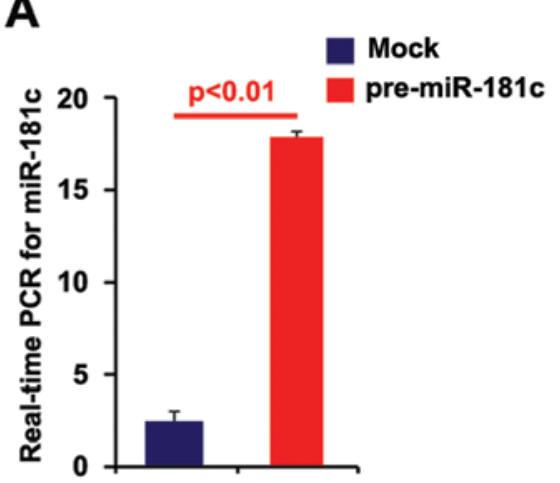

B

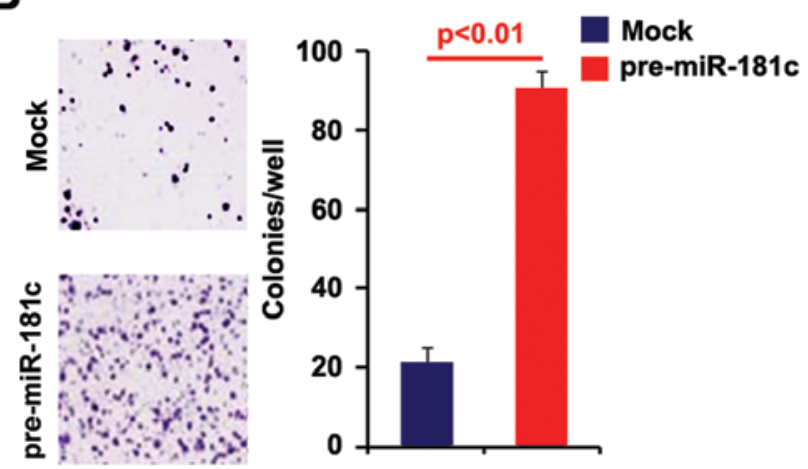

C

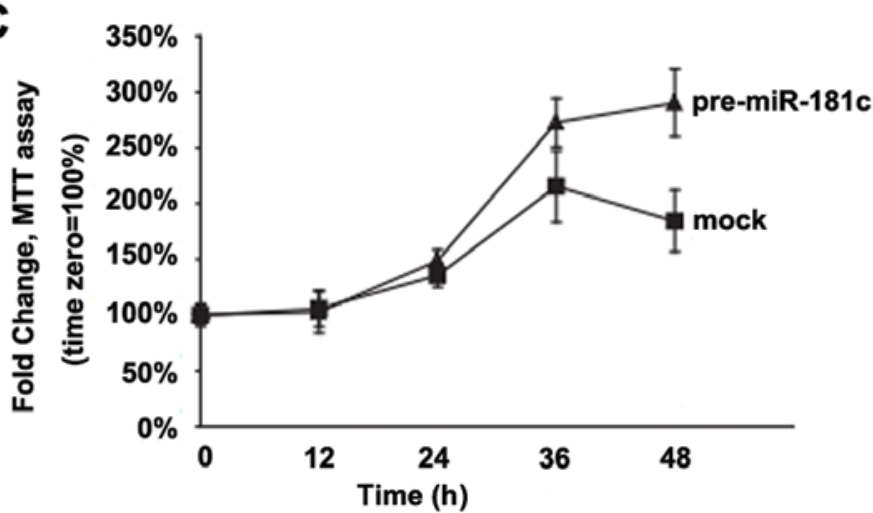

D
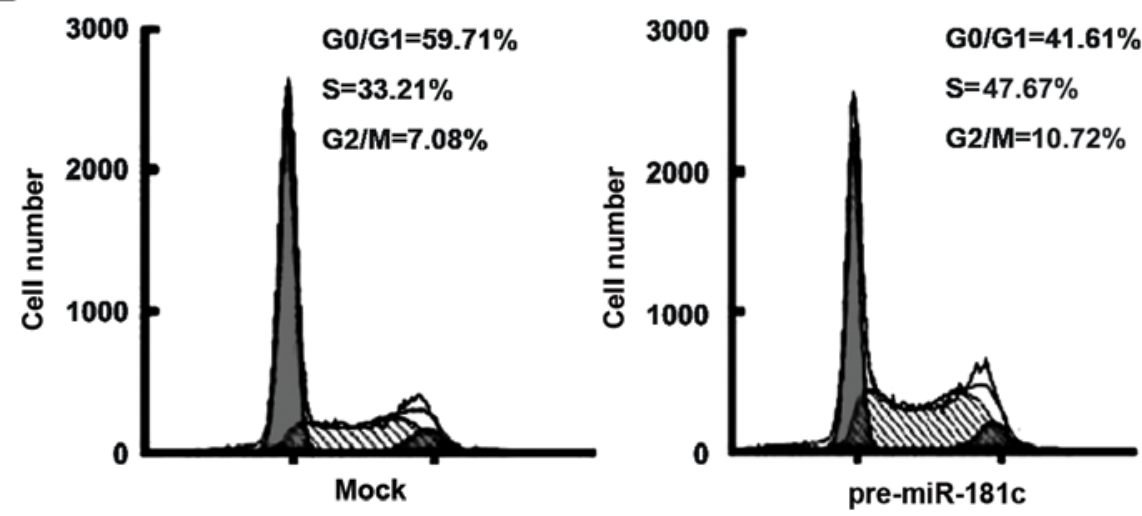

E

$\mathbf{F}$
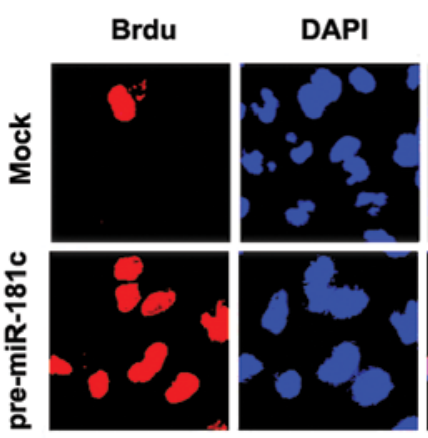

Merge
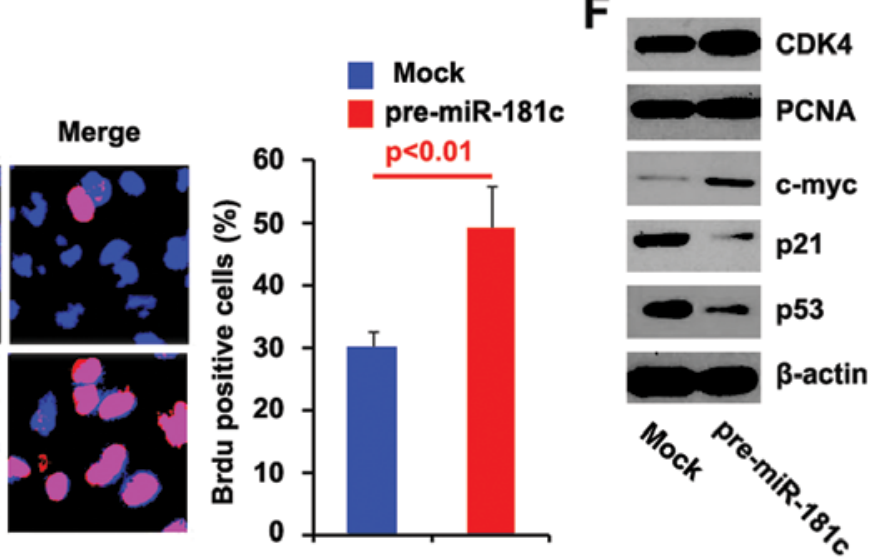

Figure 2. miR-181c promotes proliferation in inflammatory breast cancer SUM149 cells. (A) Real-time PCR for miR-181c in SUM149 cells infected with pre-miR-181c or control miR (mock). n=3. (B) Colony formation assay for SUM149 cells transfected with pre-miR-181c or control miR (mock). Colonies with $>50$ cells were counted. $n=3$. (C) MTT for SUM149 cells transfected with pre-miR-181c or control miR (mock). $n=3$. (D) Cell cycle analysis for SUM149 cells transfected with pre-miR-181c or control miR (mock). Histograms of DNA contents obtained by FACS analysis are shown. The percentages of each cell cycle stage is shown in the inset of the histograms. $\mathrm{n}=3$. (E) Brdu incorporation assay for SUM149 cells transfected as indicated. Representative micrographs (left) and quantification (right) of BrdU incorporating-cells after transfection with pre-miR-181c or control miR (mock). (F) Western blotting for CDK4, PCNA, c-myc, p21 and p53. Mock groups were transfected with control miR. $\beta$-actin was a loading control. $n=3$. 

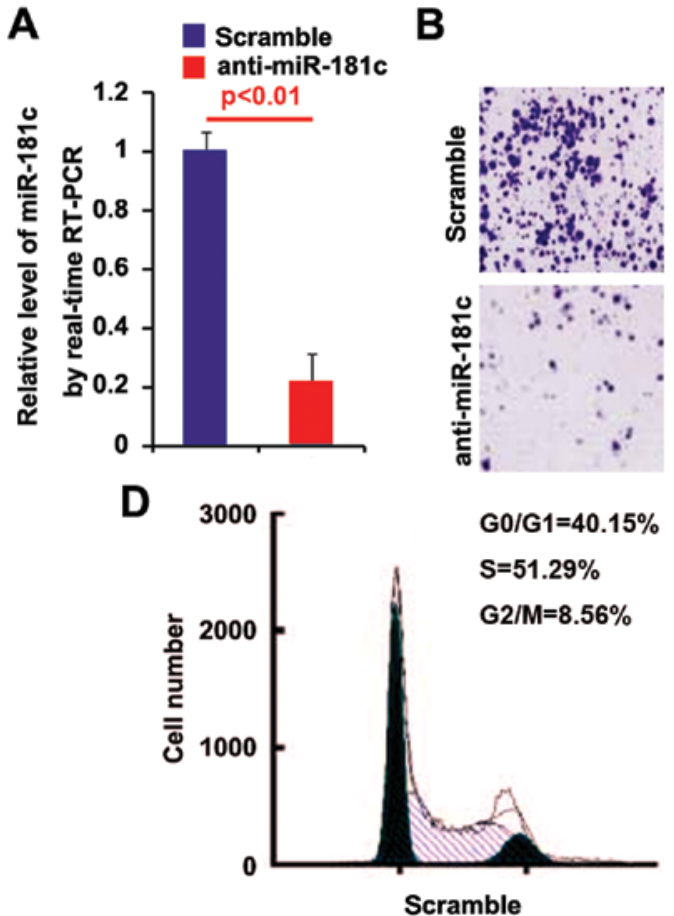

E
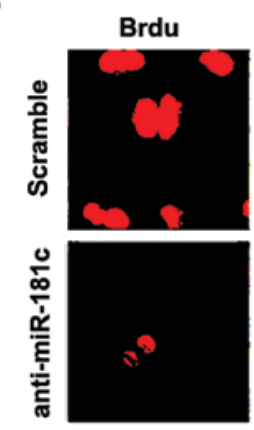

B

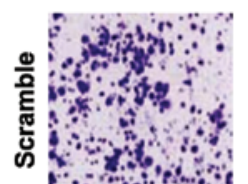

G0/G1 $=\mathbf{4 0 . 1 5 \%}$

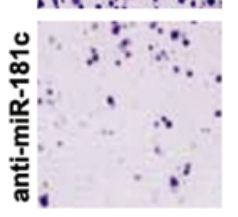

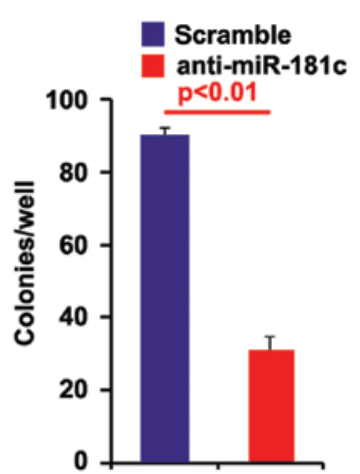

C
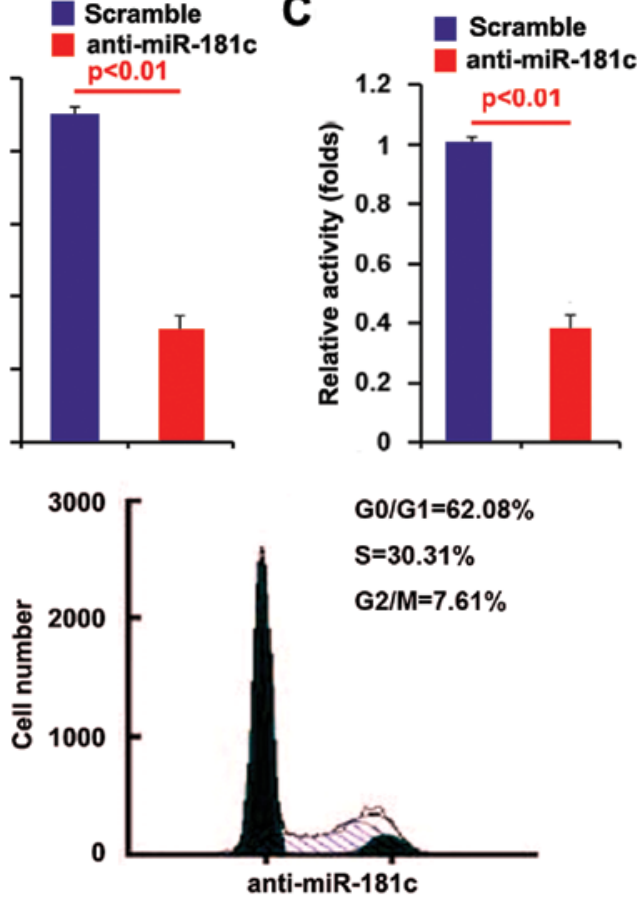

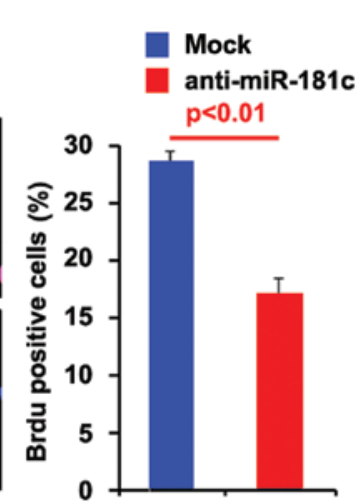

Figure 3. Sliencing miR-181c inhibits proliferation in inflammatory breast cancer SUM149 cells. (A) Real-time PCR for miR-181c in SUM149 cells infected with anti-miR-181c or scramble. $n=3$. (B) Colony formation assay for SUM149 cells transfected with anti-miR-181c or scramble. Colonies with $>50$ cells were counted. $n=3$. (C) MTT for SUM149 cells transfected with anti-miR-181c or scramble. $n=3$. (D) Cell cycle analysis for SUM149 cells transfected with anti-miR-181c or scramble. Histograms of DNA contents obtained by FACS analysis are shown. The percentage of each cell cycle stage is shown in the inset of the histograms. $n=3$. (E) Brdu incorporation assay for SUM149 cells. Representative micrographs (left) and quantification (right) of BrdU incorporating-cells after transfection with anti-miR-181c or scramble.

formation rate of SUM149 cells (Fig. 2B). Moreover, we also performed MTT assay to detect proliferation of SUM149 cells transfected with pre-miR-181c and control miR. The results showed that miR-181c promoted proliferation in SUM149 cells after $36 \mathrm{~h}$ of transfection, compared with control miR-transfected groups (Fig. 2C). To further show the effects of miR-181c on proliferation, we performed cell cycle analysis. The results showed higher $\mathrm{S}$ phase fractions in SUM149 cells transfected with pre-miR-181c than in the cells transfected with control miR (Fig. 2D). To identify that DNA synthesis promotion contributed to higher $S$ phase fractions in SUM149 cells transfected with pre-miR-181c, we performed Brdu incorporation assay to detect DNA synthesis in the cells. The results confirmed that miR-181c significantly promoted DNA synthesis in the cells and representative micrographs (left) and quantification (right) of BrdU incorporating-cells after transfection with pre-miR-181c or control miR (mock) were showed (Fig. 2E). In following studies, we performed western blotting to identify whether protein of proliferationassociated markers were also affected by miR-181c in the cells. Our studies showed that CDK4, PCNA and c-myc expression were upregulated and p21 and p53 expression were downregulated by miR-181c in the cells (Fig. 2F). These data implied that miR-181c could promote proliferation in inflammatory breast cancer.

Silencing miR-181c inhibits proliferation in inflammatory breast cancer SUM149 cells. Having demonstrated that miR181c overexpression promoted proliferation in SUM149 cells, to provide further evidence that miR-181c was involved in proliferation of SUM149 cells, we studied the effects of the inhibitor of miR-181c - anti-miR-181c. After stable transfection, miR-181c expression was detected by real-time PCR. The results showed that anti-miR-181c significantly downregulated miR-181c expression in SUM149 cells (Fig. 3A). Colony formation of SUM149 cells were tested by colony 
formation assay. Silencing miR-181c significantly suppressed colony formation of SUM149 cells (Fig. 3B). Moreover, we also performed MTT assay to detect proliferation of SUM149 cells transfected with anti-miR-181c or scramble. The results showed that anti-miR-181c inhibited proliferation in SUM149 cells, compared with scramble-transfected groups (Fig. 3C). To further show the effects of silencing miR-181c on proliferation, we performed cell cycle analysis to analyze the effect of anti-miR-181c on the cell cycle. The results showed lower S phase fractions in SUM149 cells transfected with anti-miR-181c than in SUM149 cells transfected with scramble (Fig. 3D). To identify that DNA synthesis inhibition contributed to lower S phase fractions in SUM149 cells transfected with anti-miR-181c, we performed Brdu incorporation assay to detect DNA synthesis in the cells. The results confirmed that anti-miR-181c significantly suppressed DNA synthesis in the cells and representative micrographs (left) and quantification (right) of BrdU incorporating-cells after transfection with anti-miR-181c or scramble (mock) are shown in Fig. 3E. All the results confirmed that miR-181c promoted proliferation in SUM149 cells.

miR-181c inhibited PTEN protein expression by targeting its 3'-UTR in inflammatory breast cancer. MicroRNAs (miRNAs/miRs) are a class of non-coding RNAs able to regulate gene expression at the post-transcriptional level, by binding to the 3'-untranslated region of target messenger RNAs (mRNAs) through partial sequence homology, and causing a block of translation and/or mRNA degradation (6-8). Thus, we reasoned that miR-181c inhibited proliferation by downregulating expression of specific genes. We screened targeted genes of miR-181c, using TargetScan (http://www. targetscan.org/vert_61/), and a large number of target genes were found. We were interested in PTEN, because it was regarded as an tumor suppressor gene in malignant tumors (22-24). Target sites on 3'-UTR of PTEN are shown in Fig. 4A. We reasoned that miR-181c could downregulate PTEN expression by targeting its 3'-UTR in SUM149 cells.

We performed immunofluorescence analyses in SUM149 cells transfected with pre-miR-181c or control miR. The results confirmed that PTEN protein was evidently suppressed in the cells transfected with pre-miR-181c (Fig. 4B). We next performed RT-PCR and western blotting to detect PTEN expression in SUM149 cells transfected with pre-miR-181c or control miR. The results showed that PTEN protein (Fig. 4C) was significantly downregulated in SUM149 cells transfected with pre-miR-181c, but not PTEN mRNA (Fig. 4C). Consistent with the results of RT-PCR, real-time PCR demonstrated that PTEN mRNA did not affect SUM149 cells transfected with pre-miR-181c, compared with control miR-transfected groups (Fig. 4D).

To further demonstrate the direct regulation of PTEN by miR-181c, we employed luciferase reporters with targeting sequences in 3'-UTR of PTEN (PTEN-WT-luc) (Fig. 4E). We investigated whether PTEN-WT-luc plasmids could be targeted by miR-181c in SUM149 cells. Luciferase reporter assay showed that the luciferase activity of PTEN-WT-luc plasmids was significantly suppressed in the cells transfected with pre-miR-181c, implying that miR-181c targeted 3'-UTR of PTEN mRNA (Fig. 4F). In order to further identify that
miR-181c targeted 3'-UTR of PTEN by the predicted sites, we mutated 4 bases in the predicted sites (Fig. 4E). Then mutant reporters were introduced into SUM149 cells, but the luciferase activity of PTEN-MUT-luc was not suppressed by miR-181c in SUM149 cells (Fig. 4F). Having demonstrated that miR-181c overexpression inhibited PTEN-WT-luc plasmids by the predicted sites, we next studied whether silencing miR-181c could also affect the activity of the PTEN-WT-luc plasmids. Thus luciferase reporter assay was performed and the results showed that contrary to pre-miR-181c, anti-miR-181c significantly promoted luciferase activity of PTEN-WT-luc in SUM149 cells (Fig. 4G). Moreover, mutant reporters were introduced into SUM149 cells, but the luciferase activity of PTEN-MUT-luc was not affected by anti-miR-181c in SUM149 cells (Fig. 4G). All the data illustrated that miR-181c inhibited PTEN protein expression by targeting its 3'-UTR in inflammatory breast cancer SUM149 cells.

PTEN inhibits proliferation in inflammatory breast cancer SUM149 cells. Having demonstrated that miR-181c could promote proliferation and it inhibited the tumor suppressor gene PTEN expression by targeting its 3'-UTR in SUM149 cells. We reasoned that miR-181c promoted proliferation via downregulating PTEN expression. Thus, we studied roles of PTEN in inflammatory breast cancer.

To assess the expression of PTEN in inflammatory breast cancer, western blot analysis was conducted in 6 pairs of inflammatory breast cancer tissues and matched adjacent normal tissue samples. The expression of PTEN was consistently lower in breast cancer tissues than in normal tissues (Fig. 5A). Next, we used PTEN expressing plasmids and studied roles of PTEN in SUM149 cells. In order to demonstrate that PTEN expressing plasmids could stably upregulate PTEN protein expression, western blotting was performed in SUM149 cells transfected with PTEN expressing plasmids. The results showed that PTEN protein expression was significantly increased by PTEN expressing plasmids in the cells (Fig. 5B). To identify roles of PTEN in proliferation in SUM149 cells, we performed colony formation assay, MTT assay and cell cycle analysis. Colony formation assay demonstrated that PTEN suppressed colony formation in SUM149 cells (Fig. 5C). In addition, MTT assay showed that PTEN inhibited proliferation in the cells (Fig. 5D). Consistent with colony formation and MTT assay, cell cycle assay showed that lower $\mathrm{S}$ and G2/M phase fractions in SUM149 cells transfected with PTEN than in SUM149 cells transfected with empty vector (Fig. 5E). Thus, PTEN inhibited proliferation in inflammatory breast cancer SUM149 cells.

Introduction of PTEN cDNA lacking predicted sites of 3'-UTR eliminates miR-181c cellular function. Because miR-181c directly inhibited PTEN protein expression through its 3'-UTR, we reasoned that ectopic expression of PTEN by transfection of the cDNA that did not contain the predicted target sites of 3'-UTR (in this study, the PTEN expression plasmids did not contain the predicted position in its 3 '-UTR) should escape the regulation of $\mathrm{miR}-181 \mathrm{c}$ and thus attenuate or eliminate the function of miR-181c. To this end, we transfected PTEN expressing plasmids or empty vector (pcDNA3.1) (mock) into control miR or pre-miR-181c treated SUM149 


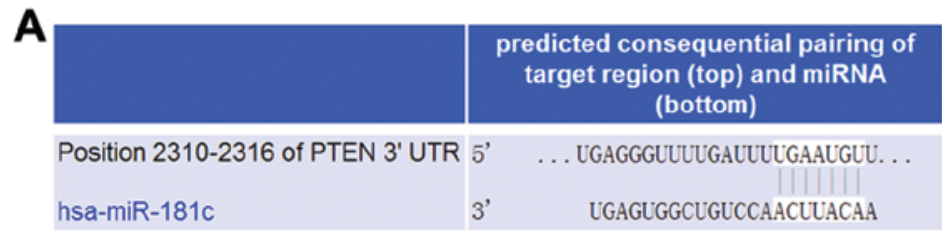

B
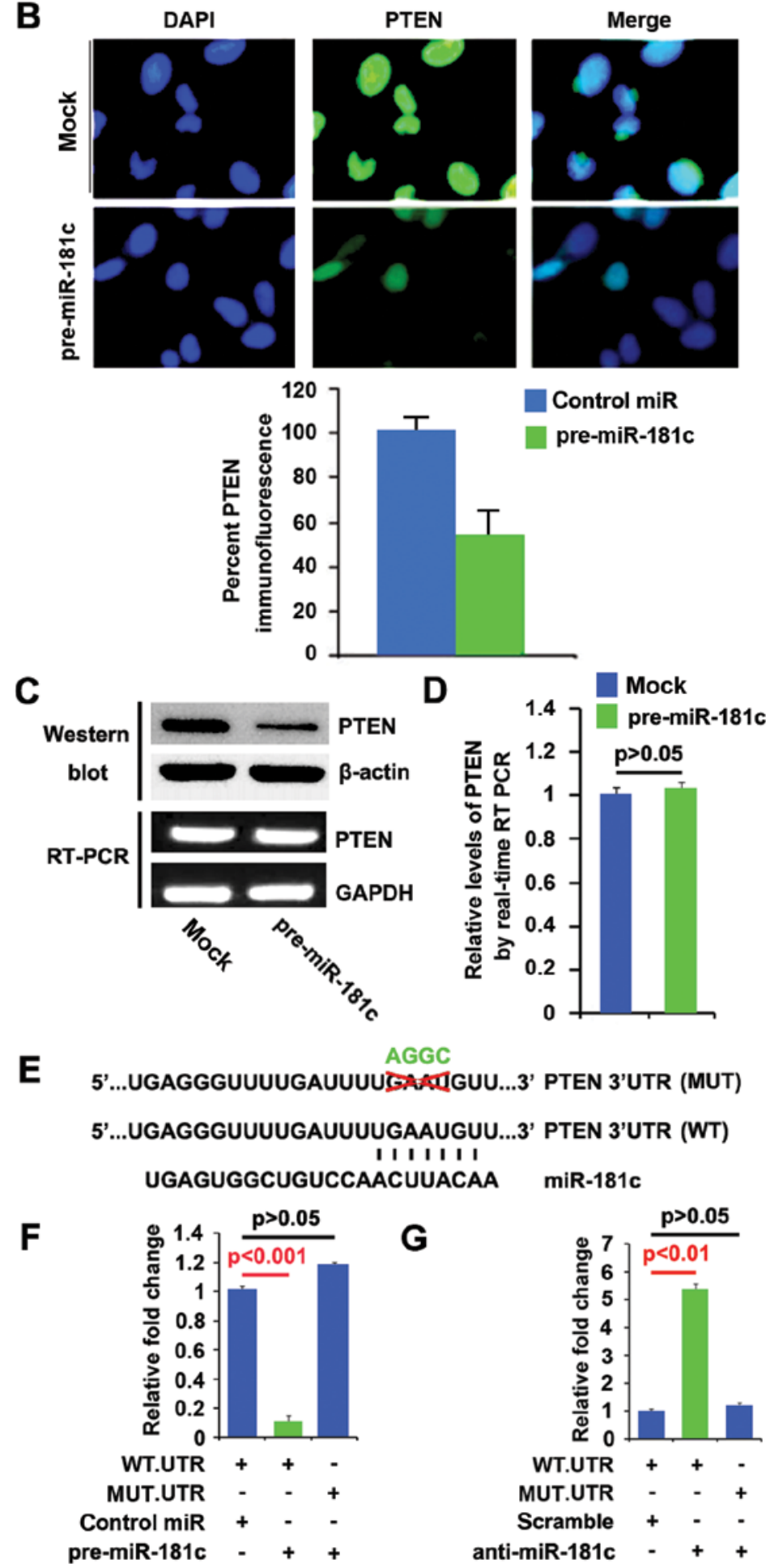

Control miR

pre-miR-181c

Figure 4. miR-181c inhibits PTEN protein expression by targeting its 3'-UTR. (A) Schematic of predicted miR-181c-binding sites in the 3'UTR of PTEN mRNA. (B) Immunofluorescence analyses of SUM149 cells transfected with pre-miR-181c or control miR (mock). Upper panel shows microscopic images of immunofluorescence staining of one representative experiment (x100 magnifications). Lower panel shows graphic presentation of mean fluorescence intensities of three independent experiments. (C) Upper panel shows western blotting for PTEN protein in SUM149 cells. Mock groups were transfected with control miR. $\beta$-actin was a loading control. Lower panel shows RT-PCR for PTEN in SUM149 cells transfected as indicated. GAPDH was a loading control. n=3. (D) Real-time PCR for PTEN mRNA in SUM149 cells infected with pre-miR-181c or control miR (mock). n=3. (E) Diagram of PTEN-3'-UTRcontaining reporter constructs. MUT, contains 4-base-mutation at the miR-181c-target region, abolishing its binding. (F) Reporter assay, with cotransfection of $500 \mathrm{ng}$ WT-or MUT-reporter and $50 \mathrm{nM}$ control-miR (mock), or pre-miR-181c as indicated. $\mathrm{n}=3$. (G) Reporter assay, with cotransfection of $500 \mathrm{ng}$ WT- or MUT-reporter and $50 \mathrm{nM}$ scramble, or anti-miR-181c as indicated. $\mathrm{n}=3$. 


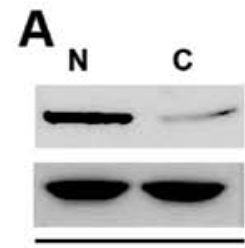

p1

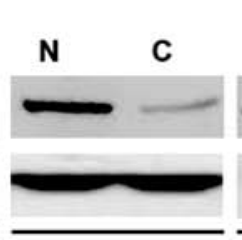

p2

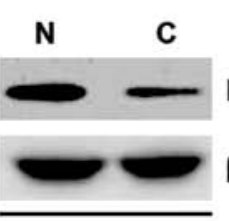

p3

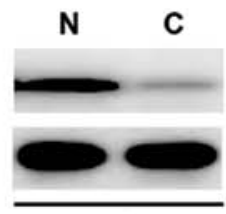

p4

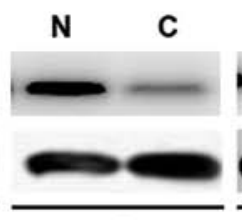

p5

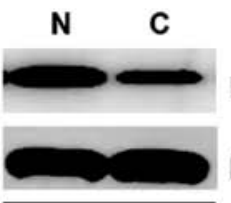

\section{PTEN}

B

PTEN

$\beta$-actin

$\beta$-actin

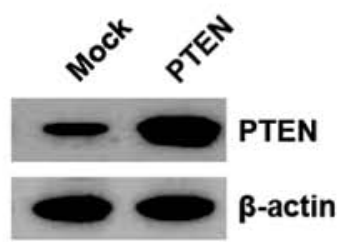

p6
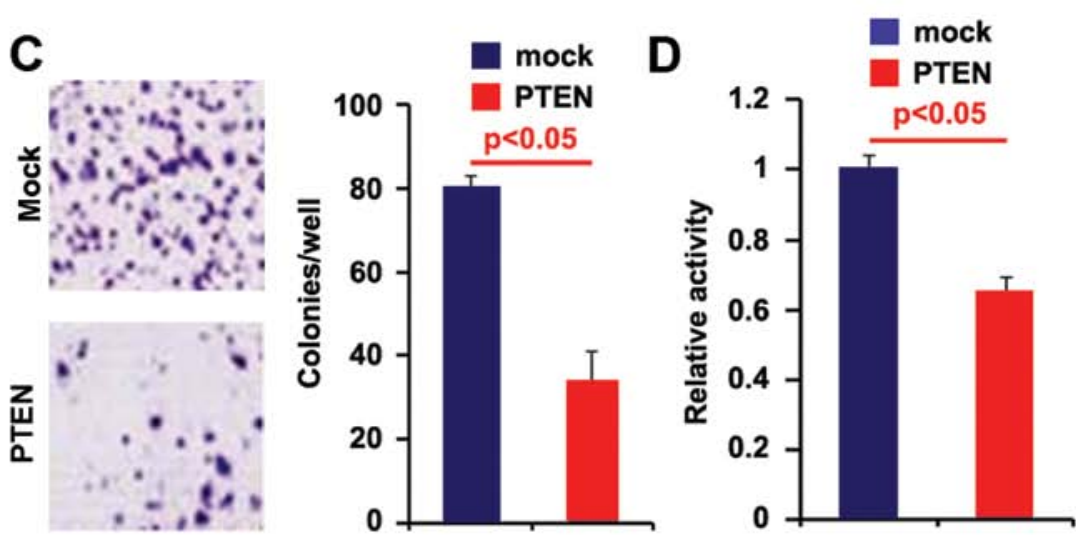

E
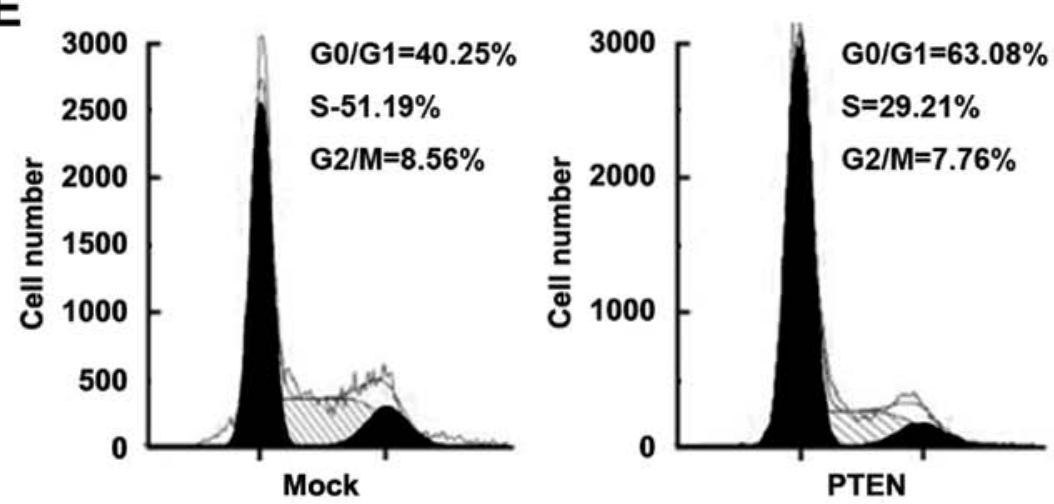

Figure 5. PTEN promotes proliferation in inflammatory breast cancer SUM149 cells. (A) Western blotting for PTEN in inflammatory breast cancer tissues and adjacent normal tissues. $\beta$-actin was a loading control. $\mathrm{n}=3$. (B) Western blotting for PTEN in PTEN expressing plasmids or empty vector (pcDNA3.1) (mock) transfected-SUM149 cells. $\beta$-actin was a loading control. $n=3$. (C) Colony formation assay for SUM149 cells transfected with PTEN expressing plasmids or empty vector (pcDNA3.1) (mock). Colonies with $>50$ cells were counted. $n=3$. (D) MTT assay for SUM149 cells transfected with PTEN expressing plasmids or empty vector (pcDNA3.1) (mock). $\mathrm{n}=3$. (E) Cell cycle analysis for SUM149 cells transfected with PTEN expressing plasmids or empty vector (pcDNA3.1) (mock). Histograms of DNA contents obtained by FACS analysis are shown. The percentage of each cell cycle stage is shown in the inset of the histograms. $\mathrm{n}=3$.

cells. Immunoblot analysis revealed that transfection of PTEN expressing plasmids attenuated the effect of miR-181c on PTEN protein (Fig. 6A).

Overexpression of miR-181c in SUM149 cells inhibited proliferation, in order to identify whether PTEN could abrogate or attenuate the roles of miR-181c on proliferation, control miR or pre-miR-181c-treated SUM149 cells were transfected with either PTEN expressing plasmids or empty vector (pcDNA3.1). We performed MTT and Brdu incorporation assay and then found that pre-miR-181c-treated SUM149 cells displayed $\sim 50 \%$ increase in proliferation than control
miR treated cells (Fig. 5B) and DNA synthesis (Fig. 5C). Overexpression of PTEN sufficed to attenuate the increase of proliferation (Fig. 5B) and DNA synthesis (Fig. 5C) observed in pre-miR-181c-treated cells. Thus, we concluded that miR-181c promoted proliferation via downregulating PTEN expression.

\section{Discussion}

MicroRNAs (miRNAs or miRs) are short non-coding RNAs that regulate the target mRNA by binding mostly to the 3'-untranslated region (3'-UTR), inducing either translational 


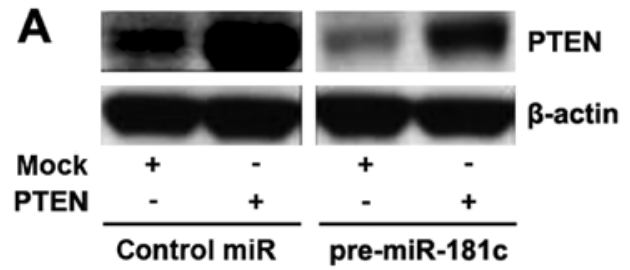

B
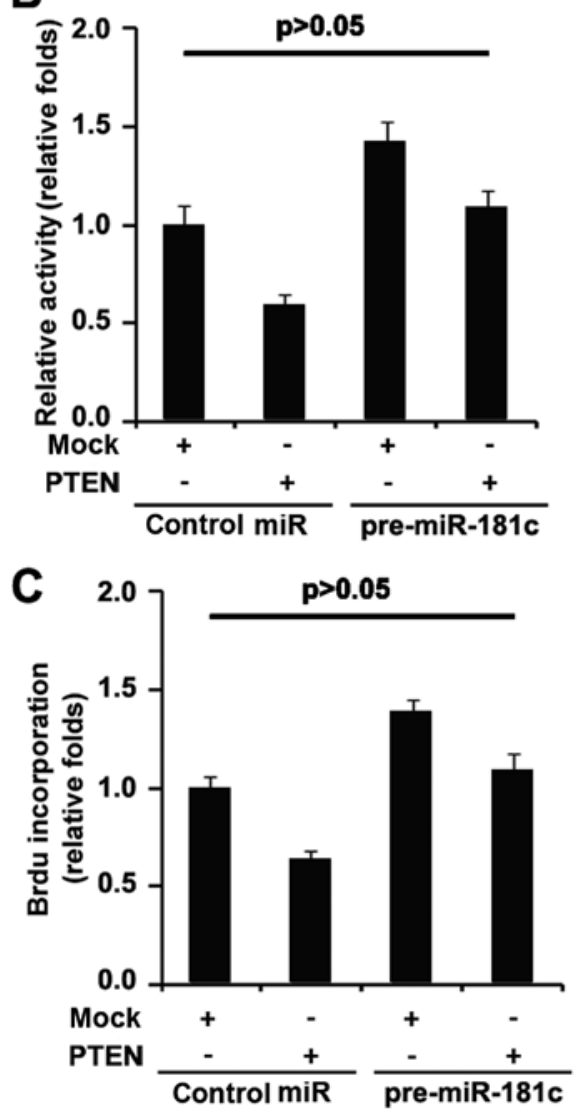

Figure 6. Introduction of PTEN cDNA lacking the predicted sites in its 3'-UTR abrogates miR-181c cellular function in inflammatory breast cancer SUM149 cells. (A) Western blotting for PTEN in pre-miR-181c or control miR treated-SUM149 cells transfected with PTEN expressing plasmids or empty vector (pcDNA3.1) (mock). $\beta$-actin was a loading control. $n=3$. (B) MTT for pre-miR-181c or control miR treated-SUM149 cells transfected with PTEN expressing plasmids or empty vector (pcDNA3.1) (mock). $n=3$. (C) Brdu incorporation analysis for pre-miR-181c or control miR treatedSUM149 cells transfected with PTEN expressing plasmids or empty vector (pcDNA3.1) (mock). $\mathrm{n}=3$.

repression or the degradation of the target (6-8). Some microRNA expression are upregulated or downregulated and function as oncogene or tumor suppressor gene by regulating specific genes in malignant tumors. For example, miR-101 promotes breast cancer cell apoptosis by targeting Janus kinase 2 (25). Upregulation of microRNA-107 induces proliferation in human gastric cancer cells by targeting the transcription factor FOXO1 (25). miR-214 was frequently deregulated in ovarian cancers and it induces cell survival and cisplatin resistance through targeting PTEN, which leads to downregulation of PTEN protein and activation of Akt pathway (26). miR-96, as a tumor suppressor gene, is strongly downregulated ( $>5$-fold) in pancreatic cancer tissues (27) and inhibits KRAS expression (28). A polycistronic microRNA cluster, miR-17-92, is overexpressed in human lung cancers and enhances cell proliferation (29). miR-10b was found to be markedly upregulated in breast cancer and tumor invasion and metastasis was initiated in disease (15). miR-184 functions as a potential oncogenic microRNA of squamous cell carcinoma of the tongue (30). miR-125b exerts tumor-suppressive effects in hepatic carcinogenesis through the suppression of oncogene LIN28B expression and suggest a therapeutic application of miR-125b in HCC (31). However, the roles of miR-181c are scarely known in malignancy.

Herein, we confirmed that miR-181c expression was upregulated in inflammatory breast cancer tissues. We studied its roles in the disease and the results showed that it could promote colony formation and proliferation in inflammatory breast cancer SUM149 cells. We also studied the mechanism of miR-181c promoting proliferation.

In the 1990s, two groups independently searching for tumor suppressors on chromosome 10q23, which is frequently deleted in advanced cancers, identified a new phosphatase termed PTEN, also known as MMAC (mutated in multiple advanced cancers) (32-34). PTEN loss of function occurs in a wide spectrum of human cancers through mutations, deletions, transcriptional silencing, or protein instability at a frequency that can rival p53 alterations in particular settings (35).

Our studies confirmed that miR-181c downregulated PTEN expression in SUM149 cells by targeting its 3'-UTR. Consistent with previous reports (32-35), we found that PTEN expression was downregulated in inflammatory breast cancer tissues, compared with normal tissues. Contrary to the role of miR-181c, it could suppress proliferation in SUM149 cells. Elucidating the mechanism that the gene is downregulated will help us to further understand the pathogenesis and progression of the disease and offer new targets for therapies. Introduction of PTEN cDNA lacking predicted sites of 3'-UTR abrogates miR-181c cellular function, suggesting that miR-181c functions as oncogene by targeting PTEN. Our results confirmed that miR-181c promoted proliferation by downregulating PTEN expression. Thus, we reason that due to miR-181c upregulation in inflammatory breast cancer, the tumor suppressive PTEN is downregulated.

miR-181c-mediated PTEN regulation in inflammatory breast cancer demonstrated in this study has potential basic and clinical implications. On the one hand, miR-181c could be a powerful oncogene by promoting proliferation and regulating relevant tumor suppressor genes in inflammatory breast cancer and pharmacological inhibition of miR-181c may represent a promising therapeutic strategy. On the other hand, PTEN is a tumor suppressor gene and inhibition of miR-181c may upregulate its expression. We are still at the beginning of the exploration of miR-181c in inflammatory breast cancer.

\section{References}

1. Key TJ, Verkasalo PK and Banks E: Epidemiology of breast cancer. Lancet Oncol 2: 133-140, 2001.

2. Lee BJ and Tannenbaum NE: Inflammatory carcinoma of the breast: A report of twenty-eight cases from the breast clinic of Memorial Hospital. Surg Gynecol Obstet 39: 580-595, 1924.

3. Jaiyesimi IA, Buzdar AU and Hortobagyi G: Inflammatory breast cancer: A review. J Clin Oncol 10: 1014-1024, 1992.

4. Cristofanilli M, Buzdar AU and Hortobágyi GN: Update on the management of inflammatory breast cancer. Oncologist 8: 141-148, 2003. 
5. Chang S, Parker SL, Pham T, Buzdar AU and Hursting SD Inflammatory breast carcinoma incidence and survival: The surveillance, epidemiology, and end results program of the National Cancer Institute, 1975-1992. Cancer 82: 2366-2372, 1998.

6. Lee RC, Feinbaum RL and Ambros V: The C. elegans heterochronic gene lin-4 encodes small RNAs with antisense complementarity to lin-14. Cell 75: 843-854, 1993.

7. Pasquinelli AE, Reinhart BJ, Slack F, et al: Conservation of the sequence and temporal expression of let-7 heterochronic regulatory RNA. Nature 408: 86-89, 2000.

8. Reinhart BJ, Slack FJ, Basson M, Pasquinelli AE, Bettinger JC, Rougvie AE, Horvitz HR and Ruvkun G: The 21-nucleotide let-7 RNA regulates developmental timing in Caenorhabditis elegans. Nature 403: 901-906, 2000.

9. Kloosterman WP and Plasterk RH: The diverse functions of microRNAs in animal development and disease. Dev Cell 11: 441-450, 2006

10. Meng F, Henson R, Wehbe-Janek H, Ghoshal K, Jacob ST and Patel T: MicroRNA-21 regulates expression of the PTEN tumor suppressor gene in human hepatocellular cancer. Gastroenterology 133: 647-658, 2007.

11. Jovanovic $M$ and Hengartner MO: miRNAs and apoptosis: RNAs to die for. Oncogene 25: 6176-6187, 2006.

12. Lu J, Getz G, Miska EA, et al: MicroRNA expression profiles classify human cancers. Nature 435: 834-838, 2005.

13. Esquela-Kerscher A and Slack FJ: Oncomirs - microRNAs with a role in cancer. Nat Rev Cancer 6: 259-269, 2006.

14. Calin GA and Croce CM: MicroRNA signatures in human cancers. Nat Rev Cancer 6: 857-866, 2006.

15. Ma L, Teruya-Feldstein J and Weinberg RA: Tumour invasion and metastasis initiated by microRNA-10b in breast cancer. Nature 449: 682-688, 2007.

16. Frankel LB, Christoffersen NR, Jacobsen A, Lindow M, Krogh A and Lund AH: Programmed cell death 4 (PDCD4) is an important functional target of the microRNA miR-21 in breast cancer cells. J Biol Chem 283: 1026-1033, 2008.

17. Yan LX, Huang XF, Shao Q, Huang MY, Deng L, Wu QL, Zeng YX and Shao JY: MicroRNA miR-21 overexpression in human breast cancer is associated with advanced clinical stage, lymph node metastasis and patient poor prognosis. RNA 14: 2348-2360, 2008.

18. Valastyan S, Reinhardt F, Benaich N, Calogrias D, Szász AM Wang ZC, Brock JE, Richardson AL and Weinberg RA: A pleiotropically acting microRNA, miR-31, inhibits breast cancer metastasis. Cell 137: 1032-1046, 2009.

19. Shimono Y, Zabala M, Cho RW, et al: Downregulation of miRNA-200c links breast cancer stem cells with normal stem cells. Cell 138: 592-603, 2009.

20. Miller TE, Ghoshal K, Ramaswamy B, Roy S, Datta J, Shapiro CL, Jacob S and Majumder S: MicroRNA-221/222 confers tamoxifen resistance in breast cancer by targeting $\mathrm{p} 27^{\mathrm{Kipl}}$. $\mathrm{J}$ Biol Chem 283: 29897-29903, 2008

21. Liao XH, Lu DL, Wang N, Liu LY, Wang Y, Li YQ, Yan TB, Sun $\mathrm{XG}, \mathrm{Hu} \mathrm{P}$ and Zhang TC: Estrogen receptor $\alpha$ mediates proliferation of breast cancer MCF-7 cells via a p21/PCNA/ E2F1-dependent pathway. FEBS J 281: 927-942, 2014
22. Estrella JS, Broaddus RR, Mathews A, Milton DR, Yao JC, Wang $\mathrm{H}$ and Rashid A: Progesterone receptor and PTEN expression predict survival in patients with low- and intermediate-grade pancreatic neuroendocrine tumors. Arch Pathol Lab Med 138: 1027-1036, 2014

23. Goschzik T, Gessi M, Denkhaus D and Pietsch T: PTEN mutations and activation of the PI3K/Akt/mTOR signaling pathway in papillary tumors of the pineal region. J Neuropathol Exp Neurol 73: 747-751, 2014

24. Nguyen HN, Yang JM, Afkari Y, Park BH, Sesaki H, Devreotes PN and Iijima M: Engineering ePTEN, an enhanced PTEN with increased tumor suppressor activities. Proc Natl Acad Sci USA 111: E2684-E2693, 2014.

25. Wang L, Li L, Guo R, et al: miR-101 promotes breast cancer cell apoptosis by targeting Janus kinase 2. Cell Physiol Biochem 34: 413-422, 2014.

26. Yang H, Kong W, He L, et al: MicroRNA expression profiling in human ovarian cancer: miR-214 induces cell survival and cisplatin resistance by targeting PTEN. Cancer Res 68: 425-433, 2008.

27. Szafranska AE, Davison TS, John J, Cannon T, Sipos B, Maghnouj A, Labourier E and Hahn SA: MicroRNA expression alterations are linked to tumorigenesis and non-neoplastic processes in pancreatic ductal adenocarcinoma. Oncogene 26: 4442-4452, 2007.

28. Yu S, Lu Z, Liu C, Meng Y, Ma Y, Zhao W, Liu J, Yu J and Chen J: miRNA-96 suppresses KRAS and functions as a tumor suppressor gene in pancreatic cancer. Cancer Res 70: 6015-6025, 2010.

29. Hayashita Y, Osada H, Tatematsu Y, Yamada H, Yanagisawa K, Tomida S, Yatabe Y, Kawahara K, Sekido Y and Takahashi T: A polycistronic microRNA cluster, miR-17-92, is overexpressed in human lung cancers and enhances cell proliferation. Cancer Res 65: 9628-9632, 2005.

30. Wong TS, Liu XB, Wong BY, Ng RW, Yuen AP and Wei WI: Mature miR-184 as potential oncogenic microRNA of squamous cell carcinoma of tongue. Clin Cancer Res 14: 2588-2592, 2008.

31. Liang L, Wong CM, Ying Q, et al: MicroRNA-125b suppressesed human liver cancer cell proliferation and metastasis by directly targeting oncogene LIN28B2. Hepatology 52: 17311740,2010

32. Steck PA, Pershouse MA, Jasser SA, et al: Identification of a candidate tumour suppressor gene, MMAC1, at chromosome $10 \mathrm{q} 23.3$ that is mutated in multiple advanced cancers. Nat Genet 15: 356-362, 1997

33. Li J, Yen C, Liaw D, et al: PTEN, a putative protein tyrosine phosphatase gene mutated in human brain, breast, and prostate cancer. Science 275: 1943-1947, 1997.

34. Li DM and Sun H: TEP1, encoded by a candidate tumor suppressor locus, is a novel protein tyrosine phosphatase regulated by transforming growth factor $\beta$. Cancer Res 57: 2124-2129, 1997.

35. Ali IU, Schriml LM and Dean M: Mutational spectra of PTEN/ MMAC1 gene: A tumor suppressor with lipid phosphatase activity. J Natl Cancer Inst 91: 1922-1932, 1999. 